\title{
A Distributed Positioning System based on Real-Time RSSI Enabling Decimetric Precision in unmodified IEEE 802.11 Networks
}

\author{
Stefano Maddio, Member, IEEE, Marco Passafiume, Alessandro Cidronali, Member, IEEE, Gianfranco \\ Manes, Member, IEEE \\ Department of Information Engineering, University of Florence, V.S. Marta, 3, I-50139, Florence, Italy \\ name. surnameeunifi.it
}

\begin{abstract}
In this paper we present a distributed positioning system for indoor environments based on a network of compact independent anchor nodes operating as specialized WiFi access points. Each node is built with component-off-the-shelves and it is capable of standard IEEE 802.11 connectivity at $2.45 \mathrm{GHz}$. The enabling technology for the localization is the Switched Beam Antenna (SBA) equipped in each node, which permits a space division multiple access at network-level.

The positional information is the result of a maximum likelihood estimation driven by the expected signal space partition of the constellation, and it is tolerant to noisy power measurements, such as Received Signal Strength Indicator, thanks to angular filtering capability of the SBA, which in addition operates in circular polarization.

Experimental validations demonstrate the performance of a 3-anchors network, operating within the IEEE 802.11 protocol, monitoring a single nomadic node inside a $7 \mathrm{~m}^{2}$ indoor square area. It results that $68 \%$ of the square area is covered with a localization error below $50 \mathrm{~cm}$, with a mean error of $47 \mathrm{~cm}$. Inside the triangular mesh defined by the three anchors, the mean error drops to $39 \mathrm{~cm}$, with $88 \%$ of the area being below $50 \mathrm{~cm}$. In addition, the maximum error is always below $77 \mathrm{~cm}$.
\end{abstract}

Index Terms-Indoor positioning system, Received Signal Strength Indicator (RSSI), Switched beam antenna.

\section{INTRODUCTION}

The interest of the scientific community to the issue of the positioning systems in GPS-denied scenarios has been in constant growth during the last years. In particular, the solutions based on simple and inexpensive Component-OffThe-Shelves (COTS) are becoming the driving force to spread economic localization systems directly available to the end users, enabling context-aware services and unattended automatic solution in many fields, such as environment monitoring [1], [2], agricultural monitoring [3], [4], prevention of art deterioration [5], [6], surveillance application [7], [8].

Among the various localization strategies, the approaches based on Received Signal Strength Indicator (RSSI) are advantageous for the cost-benefit trade-off. RSSI is a coarse but cheap power meter equipped in IEEE legacy transceivers. The most common use for the RSSI is range triangulation by the inversion of propagation models [9]. Unfortunately the complexity of the indoor radio channel leads to high range error and subsequent triangulation inaccuracy.

the RSSI measurements can also be employed in combination with Space Division Multiple Access (SDMA) [10], [11]. A convenient way to implement SBDA is by the use of Switched Beam Antennas (SBA), which are specialized radio systems capable to radiate $\mathrm{N}$ directional beam patterns, alter- natively isolating the signal reception from specific angular regions.

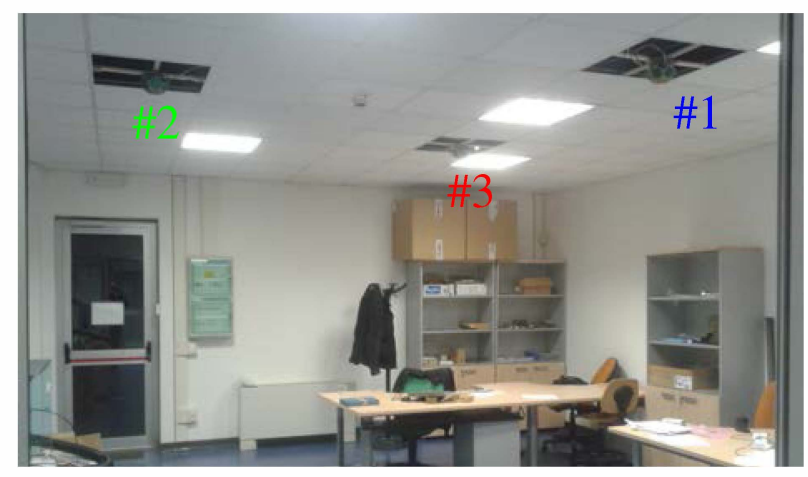

Fig. 1: Indoor test site with a constellation of 3 anchors.

With this approach, the position is estimated with the same hardware for the communication, during the normal exchange of standard WiFi messages: when a nomadic node - that could be a specific WSN node, as well as a mobile device starts a communication with underlying network through the fixed anchor nodes, which collect the RSSI for every packet detected by each antenna. The correlation of the RSSI datasets with stored power distribution of the area of interest results in a positional estimation at very low computational cost. To increase the link immunity to multi-path the SBA operates in Circular Polarization (CP) [12], [13]. Taking advantage of this mechanism, the power distribution can be estimated applying simple propagation model, such as One Slope Model (OSM) without the need of lengthy off-line setup [14], [15].

In this paper a network of 3 anchors equipped with 8-beams SBAs, designed to be placed on the ceiling, demonstrates that deci-metric localization errors are possible operating with RSSI alone. Our experimental validations in a $2.75 \mathrm{~m} \times 2.5 \mathrm{~m}$ indoor area, with furnishings and crossing people demonstrate that $90 \%$ of the room is covered with decimetric error monitoring the WiFi comunication of a single node. The perfomance of the system are summarized in a mean error of $35 \mathrm{~cm}$ with a $\sigma$ of $20 \mathrm{~cm}$ with an anchor density below $0.45 \mathrm{~m}^{-2}$.

\section{Architecture of the System}

The anchor node of the network, depicted in Fig. 3, is based on the design reported in [16], with the additional improvements investigated in [11], [17]. 


\section{A. Transceiver}

The system is based on the CC2430 System-on-Chip from Texas Instruments. The embedded transceiver is IEEE 802.15.4 and ZigBee compliant with a sensitivity of $-100 \mathrm{dBm}$.

The RSSI module operates on the received signal averaging the energy for $128 \mu \mathrm{s}$ (8 symbol periods), in accord with the IEEE protocol. This 8-bit data is related to the effective incoming power through a calibration formula.

\section{B. Switched Beam Antenna}

Switched Beam Antennas (SBA) are electronically controlled sectorized radiators. In comparison to adaptive beamformers SBA's based on RF switches offer a cost effective low-complexity solution to control the radiation patterns.

The key of space division for localization purposes consists in designing patterns with maximal angular orthogonality, hence the arrangement of the proposed node is studied to have patterns with minimal superposition [11].

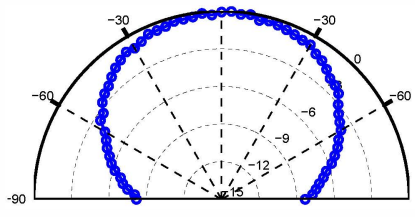

(a) Element \#2 - \#7

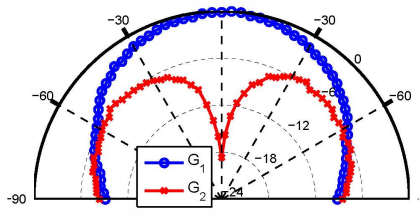

(b) Element \#1 + \#8
Fig. 2: Patterns of the elements composing the proposed SBA.

The proposed SBA arrangement, depicted in Fig. 3a, is based on a hemi-spherical 7-sided polyhedral structure, since the anchor is designed to be placed the ceiling. The cumulative pattern, i.e. the envelope of the beam set, is expected to cover an hemi-sphere without null areas. The 8 antennas assembled on this arrangement are controlled by a SP8T non reflective FET switch.

The 6 side elements, based on the design proposed in [18], are arranged with a relative dihedral angle of 110 and 120 respect to the top face, exploit the $\phi$ angle diversity exhibiting a half power angle of $80^{\circ}$ - cf Fig. 2a. A different antenna placed on the top face radiates a $\Sigma-\Delta$ pair of beams selectively activate two different pins, hence is indicated as $\# 1+\# 8$ in Fig. 2b and Fig. 3a. This behavior is advantageous to increase the discrimination along the $\theta$ direction, (cf [17]) in addition to the $\phi$-diversity exploited by each of the pairs \#1 vs $\#\{2-7\}$. The fabricated prototype is depicted in Fig. 3 b.

\section{Node Constellation}

Considering the multi-path immunity of the proposed SBA, strengthened by the $\mathrm{CP}$ operation, it is possible to estimate the power distribution of each radiation beam of the set of anchors applying a simple but non unrealistic path-loss model like One Slope Model/Friis-like model. A simple parametric analytical model for the cardioid pattern depicted in Fig. 2a can be used [10], [17]:

$$
G_{n}(\theta, \phi)=G_{n}\left[\frac{1}{2}\left(1+\cos \left(\theta-\theta_{n}, \phi-\phi_{n}\right)\right)\right]^{m_{n}}
$$

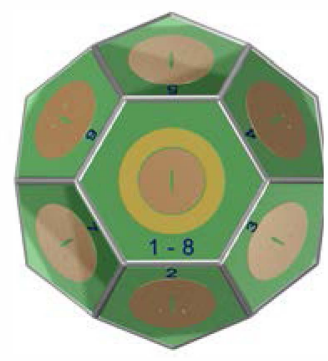

(a) Top view render

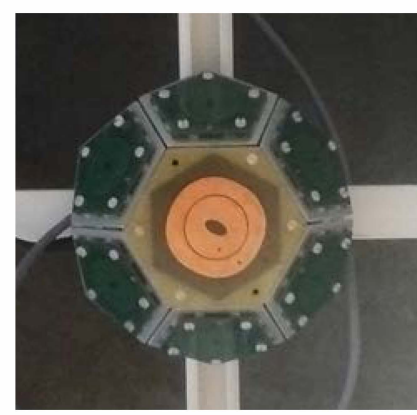

(b) Photo the actual prototype
Fig. 3: Detail of the SBA of the anchor node.

where the bi-dimensional cosine can be written as [10], [16]:

$$
\begin{aligned}
& \cos \left(\theta_{1}-\theta_{2}, \phi_{1}-\phi_{2}\right)= \\
& \quad \sin \left(\theta_{1}\right) \sin \left(\theta_{2}\right) \cos \left(\phi_{1}-\phi_{2}\right)+\cos \left(\theta_{1}\right) \cos \left(\theta_{2}\right)
\end{aligned}
$$

and $m_{n}$ is inversely proportional to half power $(3 \mathrm{~dB})$ angle according to:

$$
m=\frac{1}{\log \left(1+\cos \left(\theta_{H P}\right)\right)-1}
$$

The parameter $G_{n}, m_{n}$, and even the pointing angles $\left(\theta_{n}, \phi_{n}\right)$ can be separately adjusted to match the on of the actual beams. A slightly modified model for the antenna \#8, which presents a complementary pattern respect to antenna \#1, can be found in [17].

Figure 4 shows the partition capability of a constellation of three anchors placed down-facing at the height of 3 meter at the height. The anchor are placed at the vertex of an equilateral triangle of side $=3 \mathrm{~m}$. Focusing on the plane at the height of $1.2 \mathrm{~m}$, the typical height of an hand-held device, the area beneath the constellation is partitioned by the sectors of the multiple set of beams, one from each anchors, projected on the area of interest. The cumulative pattern in Fig. 4a covers the area an area of interest ranging from $-60 \mathrm{dBm}$ to $-44 \mathrm{dBm}$.

\section{LOCALIZATION STRATEGY}

The proposed system is based on the concept of Space Division Multiple Access as the key of the position estimation as the result of a likely-hood approach. The rough space partition induced by each antenna influence area in Fig. 4a can be considered a coarse localization technique by itself, which can play the role of pre-conditioner for the actual algorithm.

The distribution of the radiated RF signals, obtained by the projection model, are stored as power-maps, one for each anchor. With the aid of limited sets of test-points, the value of the maps can be updated modifying the parameters in (1).

Upon the sequential reception of the radio message through the beam set of each anchor, the RSSI are collected and sent to a remote node. The RSSI dataset is expected to match the following observation model:

$$
S_{n}=G_{n}(\theta(x, y), \phi(x, y))+P A T H L S S(x, y)
$$

Where $n=1, \ldots, 8$ is the antenna index of each anchor, $G_{n}(x, y)=G_{n}(\theta(x, y), \phi(x, y))$ is the angular gain 


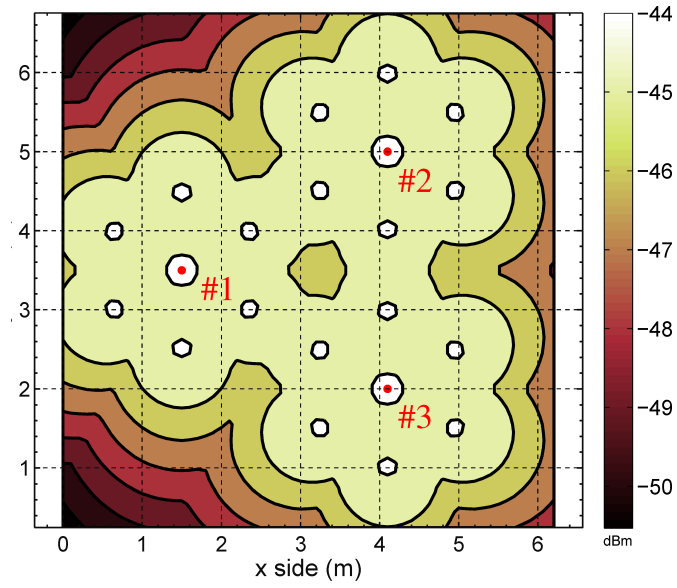

(a) Cumulative Power pattern

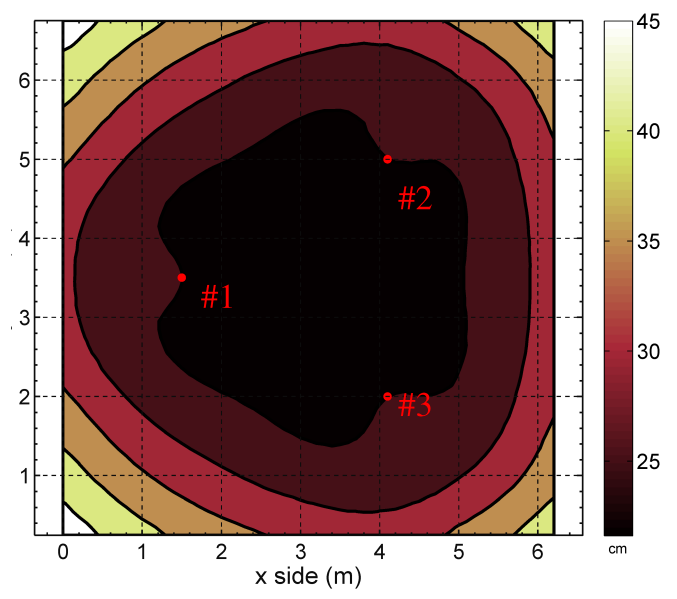

(b) CRB based on the data model

Fig. 4: Power distribution and CRB for the proposed network of anchor nodes.

remapped in the Cartesian reference - fixed the height, there is a bijective relationship between $(\mathrm{x}, \mathrm{y})$ and $(\theta, \phi)$ - and PATHLOSS $(x, y)$, is the line-of-sight propagation loss, the same for all antennas for the far-field hypothesis:

$$
\operatorname{PATHLOSS}(x, y)=10 \alpha \log 10\left(\frac{4 \pi D(x, y)}{\lambda_{0}}\right)
$$

As a side note, $\alpha$, the propagation factor equal to 2 for an exact Friis model, can be adjusted as a parameter.

The positional information is estimated through a likelihood criteria operating on the real-time RSSI data-sets collected on-line and the stored power-maps. The algorithm determines the position exploiting the pattern cross correlation approach proposed in [19], and modified in [20] to performs as a MuSiC-like algorithm. On the basis of the model in (4), it is possible to analytically estimate the minimum variance of any unbiased estimator based on the same approach. This analytical quantity, known as Cramer Rao Bound [9], [10], is depicted in Fig. $4 \mathrm{~b}$ for the constellation in exam.

The actual geometry of the anchors and their arrangement in the space has a very strong impact on this quantity, which is an inestimable tool for predicting the limit of the localization performance [21], [22].

\section{EXPERIMENTAL VALIDATION}

This section presents the experimental validation of the proposed system. An extensive measurement campaign is conducted in a laboratory of the university of Florence, a wide open space office with furniture, shown in Fig. 1. The experimental results for the area in exam are presented in Fig. 5. The three anchors, labeled with a progressive number, are hanging from the ceiling at the height of $2.7 \mathrm{~m}$, while the target node is fixed to a support at the height of $1.2 \mathrm{~m}$. Therefore the relative distance is no less than $1.5 \mathrm{~m}$, which is adequate for a far field model at $2.45 \mathrm{GHz}$.

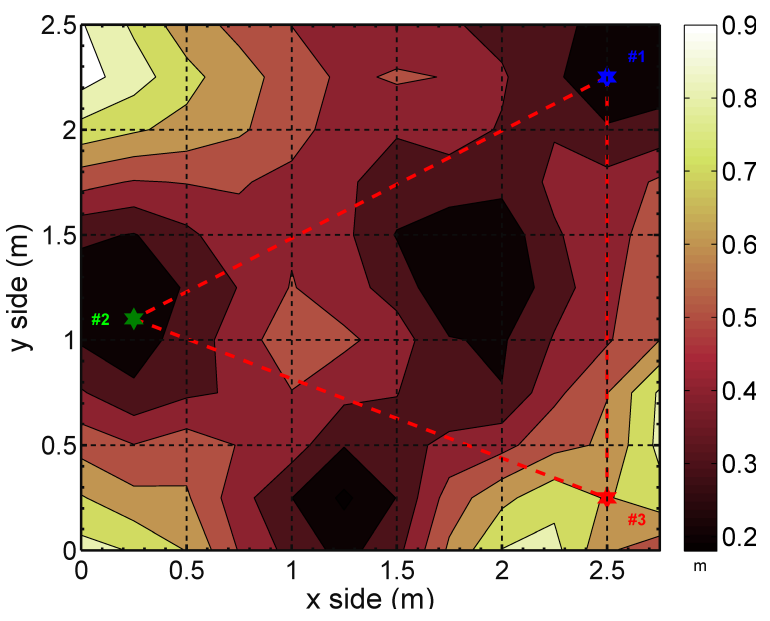

(a) mean localization error (m)

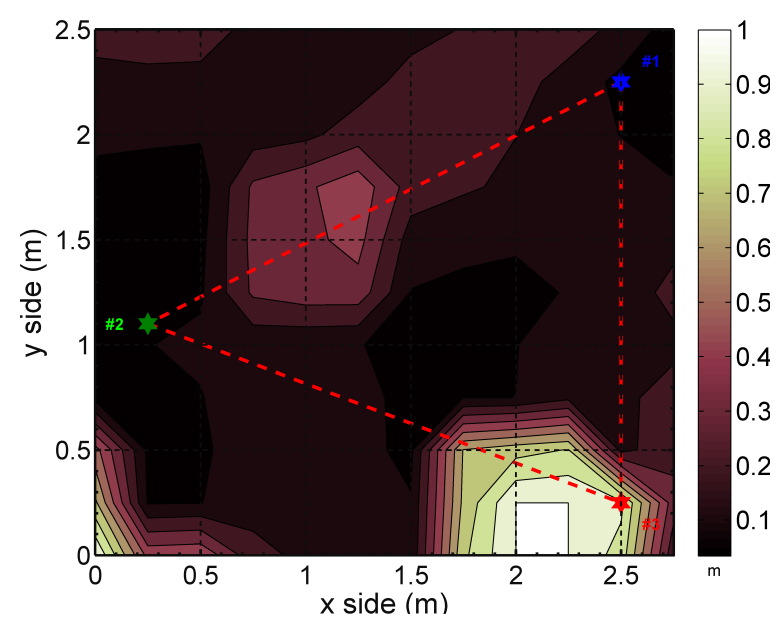

(b) localization standard deviation (m)

Fig. 5: Experiment results of the localization test.

The localization error is always sub-metrical over the square area of $7 \mathrm{~m}^{2}$, as depicted in Fig. 5a. It is worth noting how the latter matches the analysis in Fig. 4b. In particular, the error is below $50 \mathrm{~cm}$ in $62.5 \%$ of the area and below $75 \mathrm{~cm}$ in $90.8 \%$, with a mean error of $47 \mathrm{~cm}$.

Limiting the analysis on the triangular area directly covered by the mesh $\left(\approx 3 \mathrm{~m}^{2}\right)$, the localization error improves drastically with error below $50 \mathrm{~cm}$ measured in $86.5 \%$ of the area. 
TABLE I: Error coverage. All dimensions in $\mathrm{cm}$.

\begin{tabular}{l|c|c|c|c|c|c|c|c|c|c|c||c}
\hline coverage & $\min$ & $10 \%$ & $20 \%$ & $30 \%$ & $40 \%$ & $50 \%$ & $60 \%$ & $70 \%$ & $80 \%$ & $90 \%$ & $100 \%$ & mean \\
\hline \hline entire square & 18.0 & 28.7 & 32.0 & 36.9 & 41.2 & 44.9 & 49.5 & 53.8 & 61.3 & 73.6 & 94.5 & 47.5 \\
\hline within the mesh & 20.6 & 24.1 & 29.3 & 32.5 & 36.0 & 39.3 & 41.4 & 44.0 & 47.3 & 51.7 & 76.9 & 39.3 \\
\hline
\end{tabular}

A mean error of $39 \mathrm{~cm}$ is reported, with a maximum error of $77 \mathrm{~cm}$. Detailed informations about the coverage for both the cases of square and triangle regions are reported in table I as well as mean and max errors.

Finally, Fig. 5b depicts the localization standard deviations over 100 trials. It can be seen that the deviation under the mesh is really neglectable, validating the algorithm robustness against the variability of RSSI due to radio-channel unpredictability, in comparison with ranged approaches. Therefore it is correct to state that localization error is due only to powermap modeling errors, and it can be further reduced with future improvements both in the aspect of more selective SBAs and more refined algorithms.

\section{COnClusions}

A positioning system based on a network of distributed anchor nodes was briefly presented and analyzed. The system is based on a unmodified IEEE 802.11 network composed of specialized access points. The only input data of the localization is the RSSI, which is embedded in every WiFi packet and retrieved by standard legacy IEEE transceiver. The key of the localization algorithm is the space partition provided by the network of anchors, which are equipped with specialized switched beam antennas enabling the SDMA paradigm. The positioning algorithm estimates the target location of a nomadic node correlating the real-time RSSI data, collected during the normal exchange of unmodified WiFi packets, to the expected radio power distribution map stored as a data-set.

With a mesh of 3 anchors arranged in an indoor area of $7 \mathrm{~m}^{2}$ - a density of 0.42 anchors per $\mathrm{m}^{2}$ - the localization results in a mean error of $47 \mathrm{~cm}$, with $62.6 \%$ of the area covered with error below $50 \mathrm{~cm}$ and $91 \%$ below $75 \mathrm{~cm}$. The mean error is reduced to $39 \mathrm{~cm}$, if only the area below the triangular mesh is considered. In this case the maximum error is $75 \mathrm{~cm}$ and $86.5 \%$ of the mesh is below $50 \mathrm{~cm}$. A mesh of 4 or more anchors is expected to drastically improve the localization performance, since the scalable nature of the system.

\section{REFERENCES}

[1] G. Werner-Allen, K. Lorincz, M. Ruiz, O. Marcillo, J. Johnson, J. Lees, and M. Welsh, "Deploying a wireless sensor network on an active volcano," Internet Computing, IEEE, vol. 10, no. 2, pp. 18-25, 2006.

[2] N. Malhotra, M. Krasniewski, C. Yang, S. Bagchi, and W. Chappell, "Location estimation in ad hoc networks with directional antennas," in Distributed Computing Systems, 2005. ICDCS 2005. Proceedings. 25th IEEE International Conference on. IEEE, 2005, pp. 633-642.

[3] L. Bencini, S. Maddio, G. Collodi, D. Di Palma, G. Manes, and A. Manes, "Development of wireless sensor networks for agricultural monitoring," in Smart Sensing Technology for Agriculture and Environmental Monitoring. Springer, 2012, pp. 157-186.

[4] S. Mukhopadhyay, Smart Sensing Technology for Agriculture and Environmental Monitoring. Springer Verlag, 2012, vol. 146.

[5] J. Yick, B. Mukherjee, and D. Ghosal, "Wireless sensor network survey," Computer networks, vol. 52, no. 12, pp. 2292-2330, 2008.
[6] I. Akyildiz, W. Su, Y. Sankarasubramaniam, and E. Cayirci, "A survey on sensor networks," Communications magazine, IEEE, vol. 40, no. 8, pp. 102-114, 2002.

[7] T. He, S. Krishnamurthy, J. Stankovic, T. Abdelzaher, L. Luo, R. Stoleru, T. Yan, L. Gu, J. Hui, and B. Krogh, "Energy-efficient surveillance system using wireless sensor networks," in Proceedings of the 2nd international conference on Mobile systems, applications, and services. ACM, 2004, pp. 270-283.

[8] A. Arora, P. Dutta, S. Bapat, V. Kulathumani, H. Zhang, V. Naik, V. Mittal, H. Cao, M. Demirbas, M. Gouda et al., "A line in the sand: a wireless sensor network for target detection, classification, and tracking," Computer Networks, vol. 46, no. 5, pp. 605-634, 2004.

[9] R. Zekavat and R. Buehrer, Handbook of Position Location: Theory, Practice and Advances. Wiley-IEEE Press, 2011, vol. 27.

[10] S. Maddio, A. Cidronali, and G. Manes, "Smart antennas for directionof-arrival indoor positioning applications," in Handbook of Position Location: Theory, Practice, and Advances. Wiley Online Library, 2011, pp. $319-355$.

[11] S. Maddio, M. Passafiume, A. Cidronali, and G. Manes, "A scalable distributed positioning system augmenting wifi technology," in Indoor Positioning and Indoor Navigation (IPIN), 2013 International Conference on. IEEE, 2013, pp. 1-10.

[12] T. Rappaport and D. Hawbaker, "Wide-band microwave propagation parameters using circular and linear polarized antennas for indoor wireless channels," Communications, IEEE Transactions on, vol. 40, no. 2, pp. 240-245, 1992.

[13] R. Szumny, K. Kurek, and J. Modelski, "Attenuation of multipath components using directional antennas and circular polarization for indoor wireless positioning systems," in Radar Conference, 2007. EuRAD 2007. European. IEEE, 2007, pp. 401-404.

[14] R. Wahl, O. Staebler, and M. J. Gallardo, "Requirements for indoor building databases to increase the accuracy of the propagation results," in Mobile and Wireless Communications Summit, 2007. 16th IST. IEEE, 2007, pp. 1-4.

[15] A. Alhamoud, M. Kreger, H. Afifi, C. Gottron, D. Burgstahler, F. Englert, D. Bohnstedt, and R. Steinmetz, "Empirical investigation of the effect of the door's state on received signal strength in indoor environments at $2.4 \mathrm{ghz}$," in Local Computer Networks Workshops (LCN Workshops), 2014 IEEE 39th Conference on. IEEE, 2014, pp. 652-657.

[16] A. Cidronali, S. Maddio, G. Giorgetti, and G. Manes, "Analysis and performance of a smart antenna for 2.45-ghz single-anchor indoor positioning," Microwave Theory and Techniques, IEEE Transactions on, vol. 58, no. 1, pp. 21-31, 2010.

[17] S. Maddio, A. Cidronali, and G. Manes, "An azimuth of arrival detector based on a compact complementary antenna system," in Wireless Technology Conference (EuWIT), 2010 European. IEEE, 2010, pp. 249-252.

[18] _ _ "A new design method for single-feed circular polarization microstrip antenna with an arbitrary impedance matching condition," IEEE Transactions on Antennas and Propagation, vol. 59, no. 2, pp. 379-389, 2011.

[19] E. Taillefer, A. Hirata, and T. Ohira, "Direction-of-arrival estimation using radiation power pattern with an espar antenna," Antennas and Propagation, IEEE Transactions on, vol. 53, no. 2, pp. 678-684, 2005.

[20] M. Passafiume, S. Maddio, A. Cidronali, and G. Manes, "On the duality of phase-based and phase-less rssi music algorithm for direction of arrival estimation," in Proc. 3rd International Conference on Circuits, Systems, Communications, Computers and Applications (CSCCA). WSEAS, 2014, pp. 48-54.

[21] G. Giorgetti, S. Maddio, A. Cidronali, S. Gupta, and G. Manes, "Switched beam antenna design principles for angle of arrival estimation," in Wireless Technology Conference, 2009. EuWIT 2009. European. IEEE, 2009, pp. 5-8.

[22] S. Maddio, M. Passafiume, A. Cidronali, and G. Manes, "Impact of the dihedral angle of switched beam antennas in indoor positioning based on rssi," in European Radar Conference (EuRAD), 2014 11th. IEEE, 2014, pp. 317-320. 